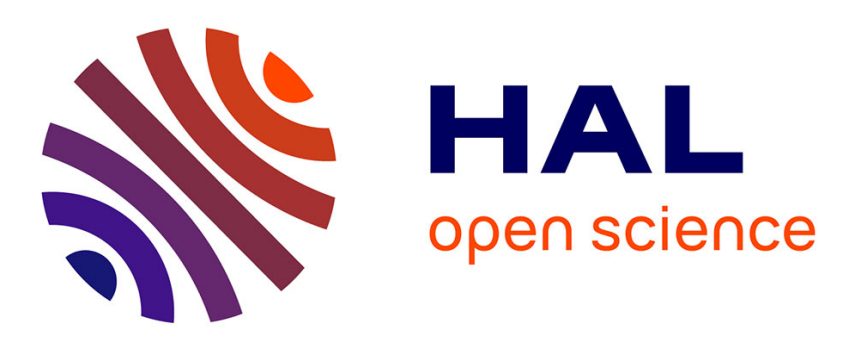

\title{
Adiabatic shear band localization fracture of solids in dynamic loading processes \\ P. Perzyna
}

\section{To cite this version:}

P. Perzyna. Adiabatic shear band localization fracture of solids in dynamic loading processes. Journal de Physique IV Proceedings, 1994, 04 (C8), pp.C8-441-C8-446. 10.1051/jp4:1994868 . jpa-00253429

\section{HAL Id: jpa-00253429 https://hal.science/jpa-00253429}

Submitted on 1 Jan 1994

HAL is a multi-disciplinary open access archive for the deposit and dissemination of scientific research documents, whether they are published or not. The documents may come from teaching and research institutions in France or abroad, or from public or private research centers.
L'archive ouverte pluridisciplinaire HAL, est destinée au dépôt et à la diffusion de documents scientifiques de niveau recherche, publiés ou non, émanant des établissements d'enseignement et de recherche français ou étrangers, des laboratoires publics ou privés. 


\title{
Adiabatic shear band localization fracture of solids in dynamic loading processes
}

\author{
P. Perzyna
}

Centre of Mechanics, Institute of Fundamental Technological Research, Polish Academy of Science, Swietokrzyska 21,00-049 Warsaw, Poland

\begin{abstract}
The main objective of the present paper is the application of a recently developed viscoplastic-damage type constitutive theory for high strain rate flow process and ductile fracture to the problem of shear band localization and fracture of dynamically loaded inelastic bodies experiencing strain rates ranging between $10^{3}-10^{4} \mathrm{~s}^{-1}$. In the first part of the paper an adiabatic inelastic flow process is formulated and investigated. The Cauchy problem is examined and the conditions for well-posedness are discussed. The relaxation time is used as a regularization parameter. The viscoplastic regularization procedure assures the unconditionally stable integration algorithm by using the finite element method. The second part of the paper is devoted to the numerical investigation of the three-dimensional dynamic adiabatic deformations of a steel thin tube twisted in a split Hopkinson bar at nominal strain rates ranging $10^{3}-10^{4} \mathrm{~s}^{-1}$.
\end{abstract}

On présente l'application d'une récente thèorie de rupture viscoplasique avec des microendommagements au problème de localization des bandes de cisaillement dans les corps non-élastiques soumis à la charge dynamique aux grands taux de déformation d'ordre $10^{3}-10^{4} \mathrm{sec}^{-1}$. Dans la première partie de l'article un processus adiabatique d'écoulement non-élastique est formulé et étudié. On formule ensuite le problème de Cauchy et on précise les conditions pour ce qu'il soit bien posé. Le temps de relaxation est utilisé comme un paramètre de régularisation. La régularisation viscoplastique assure la stabilité de l'algorithme d'intégration à l'aide de la méthode des éléments finis. La deuxième partie de l'article est consacrée à l'étude numérique d'une 3D déformation dynamique adiabatique d'un cylindre mince en acier tordu à l'aide du bar d'Hopkinson avec les taux de déformation de l'intervalle $10^{3}-10^{4} \mathrm{sec}^{-1}$.

\section{INTRODUCTION}

In technological dynamical processes fracture can occur as a result of an adiabatic shear band localization generally attributed to a plastic instability generated by thermal softening during plastic deformation.

Cho, Chi and Duffy (1988) made microscopic observations of the shear band localization on the thin-walled steel tubes in a split Hopkinson torsion bar ${ }^{1}$. Three different steels were tested. Dynamic deformation in shear was imposed to produce shear bands. It was found whenever the shear band

\footnotetext{
${ }^{1} \mathrm{Cf}$. also the investigations presented in the papers by Hartley, Duffy and Hawley (1987), Marchand and Duffy (1988) and Marchand, Cho and Duffy (1988).
} 
led to fracture of the specimen, the fracture occurred by a process of void nucleation, growth and coalescence. No cleavage was observed on any fracture surface, including the most brittle of the steel tested. This is presumably due to the thermal softening of the shear band material that results from the local temperature rise occurring during deformation process.

In recent years several investigations (e.g. Shawki and Clifton (1989), Batra (1987), Molinari and Clifton (1987) and Batra (1993)) have been taken to analyse one-dimensional simple shearing problems and particularly to study factors that infuence the initiation and development of adiabatic shear bands. Two-dimensional plane strain and axisymmetric problems have been examined numerically by Batra and Zhu (1991), Anand et al. (1987), Needleman (1989) and Batra and Ko (1992; 1993). Zbib and Jurban (1992) have investigated numerically a three-dimensional problem involving the development of shear bands in a steel bar pulled in tension and Batra and Zhang (1993) the three-dimensional dynamic thermomechanical deformations of a 4340 steel thin tube twisted in a split Hopkinson bar at nominal strain rate of 1000,2500 and $5000 \mathrm{~s}^{-1}$.

The main objective of the present paper is the application of a recently developed viscoplasticdamage type constitutive theory for high strain rate flow process and ductile fracture to the problem of shear band localization and fracture of dynamically loaded thin-walled tubes experiencing strain rates ranging between $10^{3}-10^{4} \mathrm{~s}^{-1}$. A constitutive model is developed within a thermodynamic framework of the rate type material structure with internal state variables. Such important effects as the micro-damage mechanism and thermomechanical coupling are taken into consideration.

In chapter 2 the formulation of an adiabatic inelastic flow process is given. The Cauchy problem is investigated and the conditions which guarantee its well-posedness are examined. Main feature of rate dependent plastic model have been discussed. Particular attention has been focused on the viscoplastic regularization procedure for the solution of the dynamical initial-boundary value problems with localization of plastic deformation. Some simplifications are introduced and a particular elastic-viscoplastic constitutive model for damaged solids is developed.

Chapter 3 is devoted to the numerical investigation of the three-dimensional dynamic adiabatic deformations of a steel thin tube twisted in a split Hopkinson bar at nominal strain rates ranging $10^{3}-10^{4} \mathrm{~s}^{-1}$. A thin shear band region of finite width along the circumference of the tube which undergoes significant deformations and temperature rise has been determined. Its evolution until occurrence of fracture has been simulated. Numerical results are discussed and compared with available experimental observation data.

\section{ADIABATIC INELASTIC FLOW PROCESS}

\subsection{Formulation of an adiabatic inelastic flow process}

Let us define an adiabatic inelastic flow process as follows (cf. Perzyna (1993a,b). Find $\phi, v, \rho_{M}, \tau$, $\xi$ and $\vartheta$ as function of $t$ and $\mathrm{x}$ such that

(i) the field equations

$$
\begin{aligned}
\dot{\phi}= & \boldsymbol{v} \\
\dot{\boldsymbol{v}}= & \frac{1}{\rho_{M}^{0}\left(1-\xi_{0}\right)} \operatorname{div} \tau+\frac{\boldsymbol{\tau}}{\rho_{M}(1-\xi) \rho_{M}^{0}\left(1-\xi_{0}\right)} \operatorname{grad}\left[\rho_{M}(1-\xi)\right] \\
\dot{\rho}_{M}= & -\rho_{M} \operatorname{div} \boldsymbol{v}+\frac{\rho_{M}}{1-\xi} \Xi(\mathbf{e}, \mathbf{F}, \vartheta, \xi) \\
\dot{\boldsymbol{\tau}}= & \mathcal{L}^{e}: \operatorname{sym}(D \boldsymbol{v})+2 \operatorname{sym}\left(\tau: \frac{\partial \boldsymbol{v}}{\partial \mathbf{x}}\right) \\
& -\left(\frac{\chi^{*}}{\rho_{M}(1-\xi) c_{p}} \mathcal{L}^{t h} \boldsymbol{\tau}+\mathcal{L}^{e}+\mathbf{g} \tau+\tau \mathbf{g}\right): \mathbf{P} \frac{1}{T_{m}}\left\langle\left(\frac{f}{\kappa}-1\right)^{m}\right\rangle
\end{aligned}
$$




$$
\begin{aligned}
\dot{\xi} & =\Xi(\mathbf{e}, \mathbf{F}, \vartheta, \xi), \\
\dot{\vartheta} & =\frac{\chi^{*}}{\rho_{M}(1-\xi) c_{p}} \tau: \mathbf{P} \frac{1}{T_{m}}\left\langle\left(\frac{f}{\kappa}-1\right)^{m}\right\rangle,
\end{aligned}
$$

(ii) the boundary conditions

(a) displacement $\phi$ is prescribed on a part $\partial_{\phi}$ of $\partial \phi(\mathcal{B})$ and tractions $(\boldsymbol{\tau} \cdot \mathbf{n})^{a}$ are prescribed on part $\partial_{\boldsymbol{\tau}}$ of $\partial \phi(\mathcal{B})$, where $\partial_{\phi} \cap \partial_{\boldsymbol{\tau}}=0$ and $\overline{\partial_{\phi} \cup \partial_{\boldsymbol{\tau}}}=\partial \phi(\mathcal{B})$;

(b) heat flux $\mathbf{q} \cdot \mathbf{n}=0 \Longrightarrow \operatorname{grad} \vartheta \cdot \mathbf{n}=0$ is prescribed on $\partial \phi(\mathcal{B})$;

(iii) the initial conditions

$\phi, v, \rho_{M}, \vartheta, \xi$ and $\tau$ are given at each particle $X \in \mathcal{B}$ at $t=0 ;$

are satisfied.

In the field equations (1) a mapping $\mathbf{x}=\phi(\mathbf{X}, t)$ represents a motion of a body $\mathcal{B}$, the $\operatorname{dot}$ denotes the material differentiation with respect to time $t, \mathbf{F}$ denotes the deformation gradient, $v$ is the spatial velocity field, $\tau$ the Kirchhoff stress tensor, $\rho_{M}$ and $\rho_{M}^{0}$ denote the actual and reference mass density of the matrix material, respectively, $\xi$ represents the only internal state variable and is interpreted as the porosity, $T_{m}$ dẹnotes the relaxation time for mechanical disturbances, $\mathbf{g}$ is the metric tensor in the current configuration, $D v$ denotes the spatial velocity gradient defined by

$$
(D v)_{b}^{a}=\left.v^{a}\right|_{b}=\frac{\partial v_{a}}{\partial x^{b}}+\gamma_{b c}^{a} v^{c},
$$

$\gamma_{b c}^{a}$ is the Christoffel symbol for the coordinate system $\left\{x^{a}\right\}$, the elastic matrix $\mathcal{L}^{e}$ and the thermal stress coefficients $\mathcal{L}^{\text {th }}$ are assumed in the form

$$
\left(\mathcal{L}^{e}\right)^{a b c d}=G\left(g^{a c} g^{d b}+g^{c b} g^{d a}\right)+\left(K-\frac{2}{3} G\right) g^{a b} g^{c d}+\tau^{b d} g^{a c}, \quad \mathcal{L}^{e^{-1}}: \mathcal{L}^{t h}=\theta \mathrm{g},
$$

$G$ and $K$ denote the shear and bulk modulus, respectively, $\theta$ is the thermal expansion coefficient in elastic range, the plastic potential function for damaged material is postulated in the form

$$
f=J_{2}+n \xi J_{1}^{2}, \quad \text { where } \quad J_{1}=\tau^{a b} g_{a b}, \quad J_{2}=\frac{1}{2} \tau^{i}{ }^{a b} \tau^{\prime}{ }^{c} g_{a c} g_{b d},
$$

and $n=n(\vartheta)$ is the temperature dependent material function, then

$$
P_{a b}=\frac{1}{2 \sqrt{J_{2}}} \tau^{\prime c d} g_{c a} g_{d b}+A g_{a b}, \quad A=\frac{1}{\sqrt{J_{2}}} n \xi \tau^{a b} g_{a b}
$$

the isotropic hardening-softening material function $\kappa$ is assumed in the form as follows

$$
\kappa=\kappa_{0}^{2}\left\{q+(1-q) \exp \left[-h(\vartheta) \epsilon^{p}\right]\right\}^{2}\left[1-\left(\frac{\xi}{\xi^{F}}\right)^{\frac{1}{2}}\right](1-b \vartheta),
$$

where $q=\frac{\kappa_{1}}{\kappa_{0}}, \kappa_{0}$ and $\kappa_{1}$ denote the yield and saturation stress of the matrix material, respectively, $h=h(\vartheta)$ is the temperature dependent strain hardening function for the matrix material, $\xi^{F}$ denotes the value of porosity at which the incipient fracture occurs and $b$ is a material coefficient; the overstress viscoplastic function $\Phi$ is postulated in the form (cf. Perzyna $(1963,1971)$ )

$$
\Phi(f-\kappa)=\left(\frac{f}{\kappa}-1\right)^{m}, \quad \text { where } m=1,3,5, \ldots
$$

the bracket $\langle\cdot\rangle$ defines the ramp function, $c_{p}$ is the specific heat and $\chi^{*}$ the irreversibility coefficient. 


\subsection{The Cauchy problem}

Let us consider the Cauchy problem

$$
\dot{\varphi}=\mathcal{A}(t, \varphi) \varphi+\mathrm{f}(t, \varphi), \quad t \in\left[0, t_{f}\right], \quad \varphi(0)=\varphi^{0},
$$

where $\mathcal{A}$ is a spatial differential operator and $\mathrm{f}$ is a nonlinear function, both defined by the governing equations (1), (cf. Perzyna (1993a,b)).

In order to examine the existence, uniqueness and well-posedness of the Cauchy problem (8) let us assume that the spatial differential operator $\mathcal{A}$ has domain $\mathcal{D}(\mathcal{A})$ and range $\mathcal{R}(\mathcal{A})$, both contained in a real Banach space $E$ and the nonlinear function $f$ is as follows $f: E \rightarrow E$. To investigate the existence as well as the stability of solutions to (8) it is necessary to characterize their properties without actually constructing the solutions. This can be done by considering the properties of a nonlinear semi-group because if the operator $\mathcal{A}+\mathbf{f}(\cdot)$ generates a nonlinear semi-group $\left\{\boldsymbol{F}_{t} ; t \geq 0\right\}$, then a solution to (8) starting at $t=0$ from any element $\varphi^{0} \in \mathcal{D}(\mathcal{A})$ is given by

$$
\varphi(t, \mathbf{x})=\mathbb{F}_{t} \varphi^{0}(\mathbf{x}) \quad \text { for } t \in\left[0, t_{f}\right] .
$$

We say the problem $(8)$ is well posed if $I F_{t}$ is continuous (in the topology on $\mathcal{D}(\mathcal{A})$ and $\mathcal{R}(\mathcal{A})$ assumed) for each $t \in\left[0, t_{f}\right]$.

Let us postulate as follows:

(i) the strong ellipticity condition in the form: $\mathbb{E}=\mathcal{L}^{e}$ is strongly elliptic (at a particular deformation $\phi$ ) if there is an $\varepsilon>0$ such that

$$
\mathbb{E}^{a b c d} \zeta_{a} \zeta_{c} \xi_{b} \xi_{d} \geq \varepsilon\|\zeta\|^{2}\|\xi\|^{2}
$$

for all vectors $\zeta$ and $\xi \in \mathbb{R}^{3}$;

(ii) for positive numbers $\lambda_{\mathbf{f}}^{\mathbf{l}}$ and $\lambda_{\mathbf{f}}^{2}$ and for $T_{m}>0$

$$
\mathbf{f}(t, \varphi) \in \mathrm{E}, \quad\|\mathrm{f}(t, \varphi)\|_{\mathrm{E}} \leq \lambda_{\mathbf{f}}^{1}, \quad\left\|\mathbf{f}\left(t, \varphi^{\prime}\right)-\mathbf{f}(t, \varphi)\right\|_{E} \leq \lambda_{\mathbf{f}}^{2}\left\|\varphi^{\prime}-\varphi\right\|_{E},
$$

and

$$
t \rightarrow \mathfrak{f}(t, \varphi) \in \mathrm{E} \text { is continuous. }
$$

Using the results presented by Hughes et al. (1977) and Marsden and Hughes (1983) it is possible to show (cf. Perzyna (1993a,b)) that the conditions (i) and (ii) guarantee the existence of (locally defined) evolution operators $\mathbb{F}_{t}: E \rightarrow E$ that are continuous in all variables. In other words the solution of the Cauchy problem (8) in the form (9) exists, is unique and well-posed.

\subsection{Fundamental features of rate dependent plastic model}

The conditions for the well-posedness of the Cauchy problem for the elastic-viscoplastic model of a material with softening effects have been investigated. The softening of the material is caused by the micro-damage mechanisms and thermomechanical couplings.

It has been proved that the localization of plastic deformation phenomenon in an elastic-viscoplastic solid body can arise only as the result of the reflection and interaction of waves. It has different character then that which occurs in a rate independent elasto-plastic solid body (cf. Perzyna $(1993 \mathrm{a}, \mathrm{b}))$. Rate dependency (viscosity) allows the spatial difference operator in the governing equations to retain its ellipticity and the initial value problem is well-posed. Viscosity introduces implicitly a length-scale parameter into the dynamical initial-boundary value problem and hence it implies that the localization region is diffused when compared with an inviscid plastic material. In the dynamical initial-boundary value problem the stress and deformation due to wave reflections 
and interactions are not uniformly distributed, and this kind of heterogeneity can lead to strain localization in the absence of geometrical or material irregularities. This kind of phenomenon has been recently noticed by Nemes and Eftis (1993) (cf. also the results by Sluys et al. (1992)).

The theory of viscoplasticity gives the possibility to obtain mesh-insensitive results in localization problems with respect to the width of the shear band and the wave reflection and interaction patterns (cf. Sluys et al. (1992)). Since the rate independent plastic response is obtained as the limit case when the relaxation time $T_{m}$ tends to zero (cf. Perzyna (1993a,b)) hence the theory of viscoplasticity offers the regularization procedure for the solution of the dynamical initial-boundary value problems with localization of plastic deformation.

\section{SHEAR BAND LOCALIZATION FRACTURE}

\subsection{Formulation of the initial-boundary value problem for a thin steel tube}

Cho, Chi and Duffy (1988) tested the specimens machined in the shape of thin-walled tubes with integral hexagonal flanges for gripping. Torsional loading at high strain rates was applied in a torsional Kolsky bar (split-Hopkinson bar).

We idealize the initial-boundary value problem (cf. Batra and Zhang (1993)) by assuming the specimen in the shape of thin-walled tube (the specimen dimensions are assumed the same as in the Cho, Chi and Duffy experiment). The initial conditions are taken in the form

$$
\begin{array}{rlll}
\phi(\mathrm{x}, 0)=0, & \boldsymbol{v}(\mathrm{x}, 0)=0, & & \rho(\mathrm{x}, 0)=\rho_{R e f}=\rho_{M}^{0}\left(1-\xi_{0}\right), \\
\tau(\mathrm{x}, 0)=0, & \xi(\mathrm{x}, 0)=\xi_{0}, & \vartheta(\mathbf{x}, 0)=\vartheta_{0}=\text { constant in } \mathcal{B} .
\end{array}
$$

That is, the body is initial at rest, is stress free at a uniform temperature $\vartheta_{0}$ and the initial porosity at every material point is $\xi_{0}$. For the boundary conditions, we assume

$$
\begin{aligned}
\boldsymbol{\tau} \cdot \mathrm{n} & =0 \text { on the inner and outer surfaces of the tube, } \\
\mathbf{q} \cdot \mathbf{n} & =0 \Longrightarrow \operatorname{grad} \vartheta \cdot \mathbf{n}=0 \text { on all bounding surfaces, } \\
\boldsymbol{v}\left(x_{1}, x_{2}, 0, t\right) & =0, \quad \boldsymbol{v}\left(x_{1}, x_{2}, \mathrm{~L}, t\right)=\omega^{*}(t)\left(x_{1}^{2}+x_{2}^{2}\right)^{\frac{1}{2}} \mathbf{n}^{*},
\end{aligned}
$$

where $\mathrm{n}$ is a unit outward normal to the respective surfaces, $\omega^{*}(t)$ is the angular speed of the end surface $x_{3}=\mathrm{L}$ of the tube, and $\mathbf{n}^{*}$ is a unit vector tangent to the surface $x_{3}=\mathrm{L}$. It is assumed that

$$
\omega^{*}(t)=\left\{\begin{array}{lr}
\omega_{0}^{*} t / 20, & 0 \leq t \leq 20 \mu \mathrm{s}, \\
\omega_{0}^{*}, & t>20 \mu \mathrm{s} .
\end{array}\right.
$$

The rise time of $20 \mu$ s is typical for torsional tests done in a split Hopkinson bar (cf. Batra and Zhang (1993)).

\subsection{Computation and discussion of the results}

The aforestated initial-boundary value problem has been solved by using the wide spectrum of ABAQUS possibilities (cf. Lodygowski et al. (1994)). The finite element mesh consisted by 8 -noded brick elements with 400 uniform elements along the gage length of the tube, 5 uniform across the thickness, and 100 uniform elements along the circumference.

It has been assumed following values to various material parameters (HY-100 steel)

$$
\begin{aligned}
& \rho_{M}=7860 \mathrm{~kg} / \mathrm{m}^{3}, \quad G=80 \mathrm{GPa}, \quad \vartheta_{0}=20^{\circ} \mathrm{C}, \quad \xi_{0}=0.001, \quad h=5.15, \\
& c_{p}=473 \mathrm{~J} / \mathrm{kg}^{0} \mathrm{C}, \quad K=210 \mathrm{GPa}, \quad T_{m}=5 \cdot 10^{-6} \mathrm{~s}, \quad \xi^{F}=0.25, \quad m=7, \\
& b=b^{*}\left(\frac{1}{\vartheta_{0}}-\frac{1}{\vartheta}\right), \quad b^{*}=0.01, \quad \kappa_{0}=580 \mathrm{MPa} \quad \kappa_{1}=1.2 \cdot \kappa_{0}, \quad \chi^{*}=0.85, \quad n=1.25 \text {, }
\end{aligned}
$$


The tube has been twisted at nominal strain rates ranging $10^{3}-10^{4} \mathrm{~s}^{-1}$. Particular forms of the eveluation function $\Xi$ (cf. Eqs. (1) $)_{3,5}$ ) have been postulated and discussed during the computation process. This function affects the micro-damage mechanism and has the influence on the final fracture of the specimen. It has been postulated that the intrinsic micro-damage mechanism may consist of the nucleation, growth and coalescence of microvoids (cf. Cho, Chi and Duffy (1988) experimental observations).

A thin shear band region of finite width along the circumference of the tube which undergoes significant deformations and temperature rise has been determined. Its evolution until accurrence of fracture has been simulated. It has been found that the width of the shear band region and the temperature rise vary with the nominal strain rate as well as with the relaxation time assumed. The numerical results obtained are in good agreement with experimental observation data of Cho, Chi and Duffy (1988).

An exhaustive discussion of the results obtained and the comparison with the experimental observation data of Cho, Chi and Duffy (1988) will be published elsewhere.

\section{ACKNOWLEDGMENT}

The paper has been prepared within the research programme sponsored by the Committee of Scientific Research under Grant 311129101.

\section{REFERENCES}

Anand L., Kim K.H. and Shawki T.G., J. Mech. Phys. Solids 35 (1987) 407-429.

Batra R.C., Int. J. Solids Structures 23 (1987) 1435-1446.

Batra R.C., Material Instabilities (eds. Zbib H.M., Shawki T.G. and Batra R.C), Appl. Mech. Revs. 45 (1993) S123-S131.

Batra R.C. and Ko K.I., Computational Mechs. 10 (1992) 369-379.

Batra R.C. and Ko K.I., Int. J. Engr. Sci. 31 (1993) 529-547.

Batra R.C. and Zhang X., Int. J. Plasticity (in print) (1993).

Batra R.C. and Zhu Z.G., Computers and Structures 39 (1991) 459-472.

Cho K., Chi Y.C. and Duffy J., Brown University Report (September 1988).

Hartley K.A., Duffy J. and Hawley R.H, J. Mech. Phys. Solids 35 (1987) 283-301.

Hughes T.J.R., Kato T. and Marsden J.E., Arch. Rat. Mech. Anal. 63 (1977) 273-294.

Lodygowski T., Lengnick M., Perzyna P. and Stein E., Archive of Mechanics (in print) (1994).

Marchand A., Cho K. and Duffy J., Brown University Report (September 1988).

Marchand A. and Duffy J., J. Mech. Phys. Solids 36 (1988) 251-283.

Marsden J.E. and Hughes T.J.R., Mathematical Foundations of Elasticity (Prentice-Hall, Englewood Cliffs, New York, 1983).

Molinari A. and Clifton R.J., J. Appl. Mech. 54 (1987) 806-812.

Needleman A., J. Appl. Mech. 56 (1989) 1-9.

Nemes J.A. and Eftis J., Int. J. Plasticity 9 (1993) 243-270.

Perzyna P., Quart. Appl. Math. 20 (1963) 321-332.

Petzyna P., Advances in Applied Mechanics 11 (1971) 313-354.

Perzyna P., Acta Mechanica (in print) (1993a).

Perzyna P., IUTAM Symposium on Nonlinear Waves in Solids, Victoria (Canada) 15-20 August 1993; Appl. Mech. Rev. (submitted for publication) (1993b).

Shawki T.G. and Clifton R.J., Mechanics of Materials 8 (1989) 13-43.

Sluys L.J., Block J. and de BORST R., Proc. Third International Conference on Computational Plasticity, Fundamentals and Applications, Barcelona, 6-10 April 1992 (Owen D.R.J., Onate E. and Hinton E. (eds), Pineridge Press, Swansea, 1992) pp. 539-550.

Zbib H.M. and Jurban J.S., Int. J. Plasticity 8 (1992) 619-641. 